\title{
Theoretical and experimental investigation of temperature and moisture distributions and changes in nutritional quality during Intermittent Microwave Convective Drying
}

\author{
Khan, M.I.H. ${ }^{\text {a, b}}$; Pham, N.D..$^{\text {; }}$ Karim, M.A. ${ }^{a^{*}}$ \\ a Science \& Engineering Faculty, Queensland University of Technology, \\ 2 George St, Brisbane, QLD 4000, Australia \\ bDepartment of Mechanical Engineering, Dhaka University of Engineering \& Technology \\ Gazipur-1700, Bangladesh.
}

*E-mail of the corresponding author: azharul.karim@qut.edu.au

\begin{abstract}
Intermittent microwave convective drying (IMCD) is an advanced drying system where a unique volumetric heating mode is facilitated. However, the physical phenomena of IMCD system and its effect on nutritional quality are not well understood yet. The aim of this research is to develop a coupled IMCD and quality prediction model and experimentaslly validate it. A coupled $3 D$ mathematical model considering Maxwell's equation for electromagnetic heating, and reaction kinetics for predicting quality was developed and validated. COMSOL Multiphysics, engineering software was used to solve the developed model. It is found that IMCD significantly affect the nutritional quality during drying of apple tissue.
\end{abstract}

Keywords: Food material; Microwave; heat and mass transfer; Quality; 3D modelling 


\section{Introduction}

Drying, like any other food processing methods, is a very energy intensive operation that accounts for up to $15 \%$ all industrial energy usage [1]. Understanding the coupled heat and mass transfer during drying are the dominant factor for optimising the energy and quality [2-3]. The physics-based mathematical models can provide realistic understanding of drying process.

A conventional drying or hot air drying is the easiest way of drying [4-5]. However, longer time consumption, formation of crust at the surface due to an elevated temperature, lower energy efficiency and poor quality attributes are associated with convective drying [6]. To overcome these problems, microwave drying is introduced. Microwave drying is getting popularity because it heats the sample quickly, is energy efficient, and easy to control [7]. However, the drying process using microwaves is known to yield low-quality product if not appropriately applied [8]. Hence, microwave drying has usually been combined with other drying techniques including convective hot air, vacuum and freeze-drying to achieve more uniform, fast and effective drying without significant quality loss [9]. Microwave-aided convective hot-air drying has been successfully used for some agricultural products such as grapes [10], carrot [11], apple and mushroom [12], potato[13]. However, continuous supply of microwave and convective heat may cause uneven heating or overheat or create hot spots iin the product. Heat and mass transfer should be carefully balanced to avoid such overheating and to use applied energy more efficiently [14]. Also, the quality degradation has been frequently reported during drying of foods with the continuous application of microwave energy due to uneven temperature and moisture distribution [15]. This problem can be eliminated by the application of microwave energy in a pulsed or intermittent manner that is defended as intermittent microwave convective drying (IMCD).

Intermittent microwave convective drying has proved itself an alternative method to avoid uneven heating, and to improve product quality and energy enhancement by allowing redistribution of temperature and moisture profiles within the product during off times, due to thermal diffusion [16]. Considering the benefits of IMCD drying, many researchers investigated the drying kinetics for different food samples $[8,16,17]$ experimentally or empirically. However, only experimental work cannot provide the physics for the heat and mass transfer involved in the process. Physics based mathematical modelling can provide a physical understanding of heat and mass transfer during IMWC. Malafronte, et al. [18] used multiphysics approach to model microwave-assisted convective drying. They considered heat and mass transfer and variable dielectric properties in their model. A comprehensive model for heating in microwave oven of mashed potato was developed by Chen, et al. [19]. However, these models did not consider intermittency of microwave power; thus, the temperature redistribution by means of intermittency of microwave heat source was not investigated. On the other hand, there are some single-phase models which consider the intermittency of microwave heat source [20] but these are only for microwave heating without considering mass transfer. Kumar, et al. [21] developed IMCD model using Lambert's law and observed that the predicted temperature is higher than the experimental value at the end of drying. Moreover, they pointed out that according to Lambert's law the sample surface always absorbed maximum power irrespective of moisture content, which is obviously not correct. Therefore, in this case, 
Maxwell model is the more accurate options to predict the microwave field distribution during IMCD.

In addition to this, the quality degradation is the main problem for conventional drying system. In this case, IMCD could be the best solution as it heated the sample intermittently in a periodic cycle [21]. However, the theoretical studies that deals about the effect of intermittency on quality degradation during IMCD are very rare. Therefore, the main aim of the present work is to develop a coupled 3D IMCD and quality prediction model considering the Maxwell equation for the volumetric heat generation, and reacktion kinetics for predicting the quality degradation during IMCD.

\section{Model Development}

\subsection{Governing Equations}

\subsubsection{Energy equation}

The energy balance is considered using Fourier's Law of heat transfer, as given below.

$$
\rho c_{p} \frac{\partial T}{\partial t}=\underline{\nabla} \cdot\left(k_{\text {eff }} \underline{\nabla T}\right)+\mathrm{Q}_{m}
$$

Where $\mathrm{T}$ is the temperature $\left({ }^{\circ} \mathrm{C}\right)$ at time $\mathrm{t}$, is the density of the sample $\left(\mathrm{kg} / \mathrm{m}^{3}\right)$, is the specific heat of the material $(\mathrm{J} / \mathrm{kg} / \mathrm{K})$, is the thermal conductivity of the material $(\mathrm{W} / \mathrm{m} / \mathrm{K})$, $Q_{m}$ is the volumetric heat source.

\subsubsection{Mass transfer equation}

Mass balance equation is developed based on Fick's law of diffusion that is given by,

$$
\frac{\partial c}{\partial t}+\underline{\nabla} \cdot\left(-D_{e f f} \underline{\nabla} c\right)=0
$$

where $c$ is the instantaneous moisture concentration $\left(\mathrm{mol} / \mathrm{m}^{3}\right)$, $\mathrm{t}$ is time $(\mathrm{s})$ and $D_{\text {eff }}$ is the effective moisture diffusivity $\left(\mathrm{m}^{2} / \mathrm{s}\right)$.

\subsubsection{Maxwell's equation for electromagnetics and heat generation}

Maxwell's equations provide the electromagnetic field at any point in the computational domain. In frequency domain time harmonic Maxwell's equations can be written as [22].

$\nabla \times\left(\frac{1}{\mu} \nabla \times E\right)-\frac{\omega^{2}}{c}\left(\varepsilon^{\prime}-i \varepsilon^{\prime \prime}\right) E=0$

Where, $E$ is the electric field strength, $\omega$ is the angular frequency of the microwave oven, $C$ is the speed of light, $\varepsilon^{\prime}, \varepsilon^{\prime \prime}, \mu$ are the dielectric constant, dielectric loss factor, and electromagnetic permeability of the material, respectively.

The total electromagnetics heat sources represent electromagnetic losses, $Q_{m}$, given by, [23]

$Q_{m}=Q_{r h}+Q_{m l}$

Where, $Q_{r h}$ is the resistive loss and $Q_{m l}$ is the magnetic loss. For food products, the magnetic losses are negligible, i.e. $Q_{m l}=0$.

The resistive loss can be calculated as 


$$
Q_{r h}=0.5 \cdot \vec{J} \cdot \vec{E}^{*}
$$

Where, $\vec{E}^{*}$ is the conjugate of $E$ and the electric current density $\vec{J}$ is given by

$$
\vec{J}=\sigma \cdot E=2 \pi f \varepsilon_{0} \varepsilon^{\prime \prime} \cdot E
$$

Where $f$ is the frequency of microwave, $\sigma$ is the electrical conductivity, $\varepsilon^{\prime \prime}$ is the dielectric loss factor and $\varepsilon_{0}$ is permittivity in free space.

\subsubsection{Quality prediction model}

The equation for a degradation reaction:

$-d C / d t=k . C$

Integration lead to

$C=$ Co $\exp [-k o \cdot \exp (-(\Delta E a) /(Q e \times P r+T))]$

where $\mathrm{Co}$ is the initial concentration of nutrient, $\mathrm{Pr}$ is the power ratio, and $\mathrm{ko}$ is the preexponential factor.

\section{Material and Method}

\subsection{Drying Experiment}

A new IMCD drying system has been developed to conduct the experiment, as shown in Figure 1. The IMCD system consisted of three main parts: an axial flow fan, a 3-phase 6kW heater, and a modified NN-SD691S Panasonic inverter microwave oven (2450 MHz, maximum 1100W power capacity). Moisture loss was recorded automatically by the load cell connected to a computer. The internal temperature evolution of the sample was monitored by 4 fibre optic thermal sensors which were connected to Fiber Optical 4-Channel Thermometer (OPTO con AG, Germany). The power ratio was programmed to trigger the microwave heating at the controlled condition. IMCD operations were performed at microwave power $100 \mathrm{~W}$, power ratio (on/off) of $1 / 4$, and convective temperature of $60^{\circ} \mathrm{C}$, respectively. On the completion of drying, the samples were cooled for 30 min in desiccators, wrapped with aluminium foil and store in a laboratory freezer at $-18^{\circ} \mathrm{C}$ for further analysis.

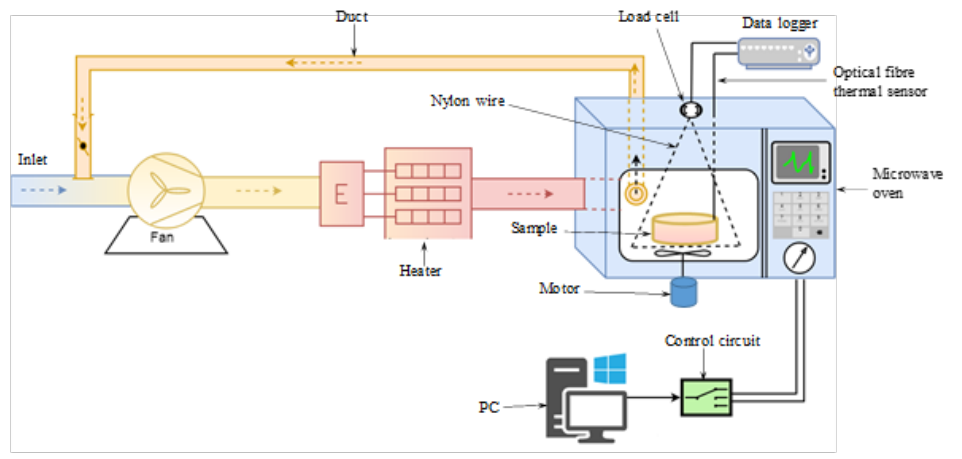

Fig. 1: Schematic diagram of the IMCD experimental setu 


\subsection{Analytical Determinations}

\subsubsection{Total phenolic extraction and content measurement}

The amount of total polyphenol in the samples were determined by the Folin-Ciocalteau method [24] with some modification. The sample was extracted in extraction solvent (methanol / distilled water $(50: 50 \mathrm{v} / \mathrm{v}))$ in amber vial at laboratory condition before homogenized in $20 \mathrm{~mL}$ of extraction solvent at maximum speed in Waring mini container blender in1 minute. Extracts were centrifuged at $15,000 \mathrm{~g}$ for 15 minutes at $20^{\circ} \mathrm{C}$. The supernatant was filtered through a Whatman no. 3 analytical filter paper. Then $0.5 \mathrm{ml}$ of the diluted extract solution was mixed with $2.5 \mathrm{ml}$ Folin- Ciocalteau reagent (10\%) and allowed 5 minutes to react, then added $0.5 \mathrm{ml}$ of $7.5 \%$ $\mathrm{Na} 2 \mathrm{CO} 3$ solution. After 30 minutes of incubation in a water bath at $37^{\circ} \mathrm{C}$, the absorbance was measured against water at $765 \mathrm{~nm}$ by Cary $50 \mathrm{UV}$ Spectrophotometer.

\subsubsection{HPLC analysis of ascorbic acid (Vitamin C)}

The sample was extracted in extraction solvent ( $3 \%$ metaphosphoric acid, $1 \mathrm{mM}$ Na2EDTA). Extracts were centrifuged at $15,000 \mathrm{~g}$ for 15 minutes. The supernatant was filtered through a Whatman no. 3 filter paper. Extraction processes were repeated three times.Vitamin C contents were determined based on Asami et al's [25] HPLC method with some modifications. The analysis was carried out using an Dionex UHPLC RS3000 system. The reverse-phase separation was obtained using a Waters Symmetry C18 column $(4.6$ x $250 \mathrm{~mm}, 5 \mu \mathrm{m})$. The isocratic mobile phase was HPLC graded water with $1 \mathrm{mM}$ EDTA and $25 \mathrm{mM}$ sodium acetate buffer acidified to $\mathrm{pH} 4.25$ with o-phosphoric acid.

\section{Results and Discussion}

\subsection{Drying Kinetics}

Average moisture content of the sample throughout the whole drying time was calculated and validated with the extensive experimental results, as shown in Figure 4. It is found that the simulated moisture content is consistent with the experimental data. To find the accuracy of the predicted data, the goodness of Fit $\left(R^{2}\right)$ was calculated and found that the value of $R^{2}$ is 0.975 . This precise R2 value indicates the developed model is quite accurate and able to predict the moisture distribution of plant-based food material during IMCD. For better interpretation about the moisture level throughout the IMCD process, 2D moisture concentration profile were drawn, as shown in Figure 5. It can be seen that the initial moisture concentration for a fresh apple tissue is uniformly distributed in the whole sample (Figure 5a). The moisture concentration decreases while drying is in progress (Figure $5 \mathrm{~b}$-e). Interestingly, it can be observed that the moisture concentration is higher at the left side of the sample as compared to the right side. This is mainly due to the non-uniform microwave energy although the temperature distribution remain almost uniform, as shown in Figure 6.

Figure 6 shows the average temperature distribution during IMCD drying. It can be seen the experimental results are closely matched with the simulated results. The dispersion pattern (fluctuation) of this figure indicates the tempering and heating period during IMCD drying. The different peak points indicate the heating time (microwave on time) and the various nadir points mean tempering time. During the IMCD, microwave heats the sample rapidly for few seconds (20s) and then it was stopped automatically for a period (e.g 60s) for completing on cycle (80s). 


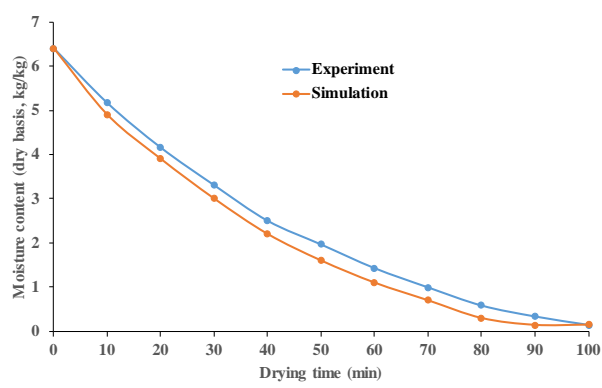

Fig. 4 The average moisture content during IMCD drying

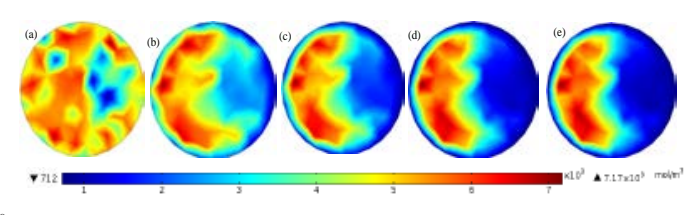

Fig 5: The concentration distribution at different stages of IMCD (a) at 0 min (b) at 20 min (c) at $40 \mathrm{~min}$ (d) at $80 \mathrm{~min}$, and (e) at 100 $\min$

The frequent microwave stop allows a time to redistribute the heat energy within the sample properly, prevents the material from overheating and therefore the better quality of the product (discussed in section4.2) can be maintained. The trend of the temperature distribution remains almost similar throughout the whole drying processes although a little attenuation can be observed at the final stages of drying. This temperature distribution mainly depends on the moisture concentration at different stages of drying (Figure 5) because microwave mainly heat firstly where it gets more moisture. At the final stages of drying, most of the water has been transported by continuous evaporation due the IMCD process progress simultaneously. As a result, most of the sample become dry, and hence the microwave cannot generate more heat. Due to this reason, the temperature decreases at the final stages of drying.

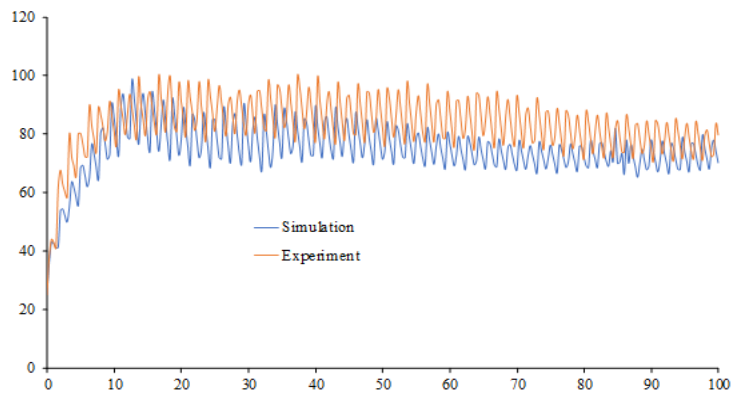

Fig. 6: The average temperature distribution during IMCD drying

\subsection{Reaction Kinetics}

Understanding of IMCD process on important quality parameters such as ascorbic acid (AA) and total polyphenol content (TPC) is an important issue due to their thermos labile sensitivities and should be taken into account in the drying process. Initial values of AA and TPC were found to be 4.842 (mg/100g fresh weight) and 70.74 (mg/100g fresh weight), respectively in a fresh granny smith apple. Figures 7 and 8 represent the degradation kinetics of AA and TPC in the Granny Smith apple slice. It can be seen that the retention remaining AA, and TPC values were reduced with drying time, and the degradation rate accelerated with increasing temperature and microwave 
power density, meaning that the loss of bioactive compounds increased. This study found that, at the beginning of the process, the effect of moisture content on the reaction rate of AA degradation seems to be predominant, while the temperature effect becomes major as the process proceeds. The degradation rate of AA was low at the beginning of the IMCD process (Figure 7) with the reduction of moisture content from $86 \%$ to $60 \%$ (Figure 4 ). When moisture content reached 55$60 \%$, the rate of this reaction reached a maximum value and at moisture contents below $55 \%$, the rate decreased significantly (Figure 7) with moisture reduction. This rapid degradation phenomenon can be attributed to the destruction of cell structure under microwave heating as it can lead to ascorbic acid release and contribute to the rapid oxidation of ascorbic acid.

Considering the evolution of ascorbic acid retention during the drying process in the present paper (Figure 7), experimental data were fitted to a first-order kinetic model. The natural logarithmic ratio of nutrient retention (ln $\mathrm{C} / \mathrm{C} 0$ ) versus drying time representation revealed linear correlations with the degradation rate constant $(\mathrm{k})$ and coefficients of determination $(\mathrm{R})$ higher than 0.98 (Figure 8), suggesting that the model was suitable in describing the degradation of ascorbic acid during IMCD drying of granny smith apples.

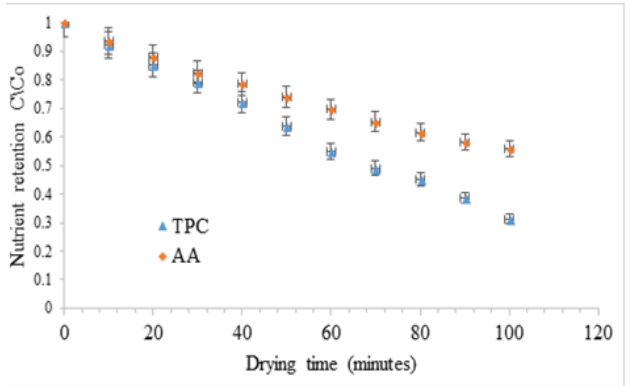

Fig. 7 Kinetics of ascorbic acid and total polyphenol alteration of Granny Smith apple during IMCD

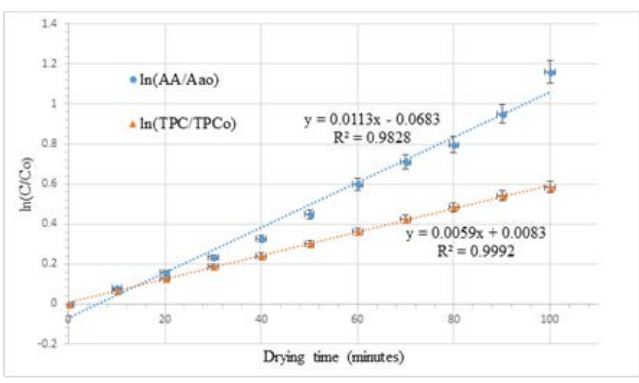

Fig 8: Predicted first-order kinetics of retention of nutrient during IMCD drying Granny Smith apple

In addition, apple is a good source of total polyphenol, which can be affected by temperature, oxygen, $\mathrm{pH}$, metal, the release of polyphenol oxidase (PPO) and other parameters. It can be seen from Figure 7, the TPC degrads highly at the first 40 minutes of IMCD, and extends even beyond the first drying period corresponded to the activation of PPO enzyme and rapid linear decrease of free moisture during IMCD drying. Then TPC gradually decreases over the vast majority of the time to reach the final stage where degradation is found to be marginal. Finally, between the two key nutrients in apple tissue, the degradation rate of ascorbic acid is higher than total polyphenol. This is may be due to higher thermal sensitivity of ascorbic acid as compared to TPC.

\section{Conclusion}

In this research, an IMCD model for food drying has been developed to predict the moisture and temperature distribution and its effect on quality of apple tissue. A 3D model has been developed considering Maxwell equations to generate volumetric heating and reaction kinetics to predic the quality degradation during IMCD drying. The developed model was then validated with the experimental results. It has been found that the predicted moisture and temperature distribution results is fully consistent with the experimental results. The precise goodness of fit $\left(R^{2}\right)$ value 
Theoretical and experimental investigation of temperature and moisture distributions and changes in nutritional quality during Intermittent Microwave Convective Drying

indicates the developed model is quite accurate and able to predict the moisture distribution of plant-based food material during IMCD. It is also found that the IMCD significantly alter two different key nutrient qualities: ascorbic acid and total polyphenol content of apple tissue. Between these two nutrients, ascorbic acid has the highest tendency to alter its character during IMCD as compared to total polyphenol content.

\section{References}

[1] Beedie, M., Energy saving-a question of quality. Dairy Industries International 1995, 60 (12), 27.

[2] Khan, M. I. H.; Kumar, C.; Joardder, M. U. H.; Karim, M. A., Determination of appropriate effective diffusivity for different food materials. Drying Technology 2017a, 35 (3), 335-346.

[3] Khan, M. I. H., Joardder, M. U. H., Kumar, C., \& Karim, M. A. (2018). Multiphase porous media modelling: A novel approach to predicting food processing performance. Critical reviews in food science and nutrition, 58(4), 528546.

[4] Joardder, M. U., Brown, R. J., Kumar, C., \& Karim, M. A., Effect of cell wall properties on porosity and shrinkage of dried apple. International Journal of Food Properties, 2015, 18(10), 2327-2337.

[5] Joardder, M. U., Kumar, C., Brown, R. J., \& Karim, M. A., A micro-level investigation of the solid displacement method for porosity determination of dried food. Journal of Food Engineering, 2015, 166, 156-164.

[6] Chou, S. K. C., K. J., New hybrid drying technologies for heat sensitive foodstuffs. Trends in Food Science \& Technology 2001, 12 (10), 359-369.

[7] Datta, A. K.; Ni, H., Infrared and hot-air-assisted microwave heating of foods for control of surface moisture. Journal of Food Engineering 2002, 51 (4), 355-364.

[8] Soysal, Y.; Ayhan, Z.; Eştürk, O.; Arıkan, M. F., Intermittent microwave-convective drying of red pepper: Drying kinetics, physical (colour and texture) and sensory quality. Biosystems Engineering 2009, 103 (4), 455-463.

[9] Wang, J.; Xiong, Y.-S.; Yu, Y., Microwave drying characteristics of potato and the effect of different microwave powers on the dried quality of potato. Eur Food Res Technol 2004, 219 (5), 500-506.

[10] Tulasidas, T. N.; Raghavan, G.S.V.; Norris, E.R., Microwave and convective drying of grapes. Transactions of the ASAE 1993, 361861-1865.

[11] Prabhanjan, D. G.; Ramaswamy, H. S.; Raghavan, G. S. V., Microwave-assisted convective air drying of thin layer carrots. Journal of Food Engineering 1995, 25 (2), 283-293.

[12] Funebo, T.; Ohlsson, T., Microwave-assisted air dehydration of apple and mushroom. Journal of Food Engineering 1998, 38 (3), 353-367.

[13] Khraisheh, M. A. M.; McMinn, W. A. M.; Magee, T. R. A., A multiple regression approach to the combined microwave and air drying process. Journal of Food Engineering 2000, 43 (4), 243-250.

[14] Gunasekaran, S., Pulsed microwave-vacuum drying of food materials. Drying Technology 1999, 17 (3), 395-412.

[15] Yongsawatdigul, J.; Gunasekaran, S., Microwave-vacuum drying of cranberries: part ii. quality evaluation. Journal of Food Processing and Preservation 1996, 20 (2), 145-156.

[16] Gunasekaran, S., Pulsed microwave-vacuum drying of food materials. Drying Technology 1999, 17, 395-412.

[17] Beaudry, C.; Raghavan, G. S. V.; Rennie, T. J., Microwave Finish Drying of Osmotically Dehydrated Cranberries. Drying Technology 2003, 21 (9), 1797-1810.

[18] Malafronte, L.; Lamberti, G.; Barba, A. A.; Raaholt, B.; Holtz, E.; Ahrné, L., Combined convective and microwave assisted drying: Experiments and modeling. Journal of Food Engineering 2012, 112 (4), 304-312.

[19] Chen, J.; Pitchai, K.; Birla, S.; Negahban, M.; Jones, D.; Subbiah, J., Heat and mass transport during microwave heating of mashed potato in domestic oven--model development, validation, and sensitivity analysis. J Food Sci 2014, 79 (10), E1991-2004.

[20] Gunasekaran, S.; Yang, H.-W., Optimization of pulsed microwave heating. Journal of Food Engineering 2007, 78 (4), $1457-1462$.

[21] Kumar, C.; Joardder, M. U. H.; Farrell, T. W.; Millar, G. J.; Karim, M. A., Mathematical model for intermittent microwave convective drying of food materials. Drying Technology 2016, 34 (8), 962-973.

[22] Chen, J.; Pitchai, K.; Birla, S.; Negahban, M.; Jones, D.; Subbiah, J., Heat and Mass Transport during Microwave Heating of Mashed Potato in Domestic Oven - Model Development, Validation, and Sensitivity Analysis. Journal of Food Science 2014, 79 (10), E1991-E2004.

[23] Wentworth, S. M., Fundamentals of Electromagnetics with Engineering Applications. John Wiley: 2004.

[24] Socha, R.; Juszczak, L.; Pietrzyk, S.; Fortuna, T., Antioxidant activity and phenolic composition of herbhoneys. Food Chemistry 2009, 113 (2), 568-574.

[25] Asami, D. K.; Hong, Y.-J.; Barrett, D. M.; Mitchell, A. E., Comparison of the Total Phenolic and Ascorbic Acid Content of Freeze-Dried and Air-Dried Marionberry, Strawberry, and Corn Grown Using Conventional, Organic, and Sustainable Agricultural Practices. Journal of Agricultural and Food Chemistry 2003, 51 (5), 1237-1241. 